\title{
LARGE SETS WITH SMALL INJECTIVE PROJECTIONS
}

\section{Frank Coen, Nate Gillman, Tamás Keleti, Dylan King and Jennifer Zhu}

Villanova University, Department of Mathematics \& Statistics 800 Lancaster Ave, Villanova, PA 19085, U.S.A.; fcoen@villanova.edu

Brown University, Department of Mathematics

Providence, Rhode Island 02912, U.S.A.; ngillman@brown.edu

Eötvös Loránd University, Institute of Mathematics

Pázmány Péter Sétány 1/c, H-1117 Budapest, Hungary; tamas.keleti@gmail.com

Wake Forest University, Department of Mathematics \& Statistics

Winston-salem, NC 27109, U.S.A.; kingda16@wfu.edu

University of California, Department of Mathematics, Berkeley

Berkeley, CA 94720, U.S.A.; jzhu42@gmail.com

\begin{abstract}
Let $\ell_{1}, \ell_{2}, \ldots$ be a countable collection of lines in $\mathbf{R}^{d}$. For any $t \in[0,1]$ we construct a compact set $\Gamma \subseteq \mathbf{R}^{d}$ with Hausdorff dimension $d-1+t$ which projects injectively into each $\ell_{i}$, such that the image of each projection has dimension $t$. This immediately implies the existence of homeomorphisms between certain Cantor-type sets whose graphs have large dimensions. As an application, we construct a collection $E$ of disjoint, non-parallel $k$-planes in $\mathbf{R}^{d}$, for $d \geq k+2$, whose union is a small subset of $\mathbf{R}^{d}$, either in Hausdorff dimension or Lebesgue measure, while $E$ itself has large dimension. As a second application, for any countable collection of vertical lines $w_{i}$ in the plane we construct a collection of nonvertical lines $H$, so that $F$, the union of lines in $H$, has positive Lebesgue measure, but each point of each line $w_{i}$ is contained in at most one $h \in H$ and, for each $w_{i}$, the Hausdorff dimension of $F \cap w_{i}$ is zero.
\end{abstract}

\section{Introduction and statement of results}

Weierstrass famously constructed a function which is everywhere continuous but nowhere differentiable. The so-called Weierstrass function is defined in his original 1872 paper [9] as the following Fourier series,

$$
f(x)=\sum_{n \geq 0} a^{n} \cos \left(b^{n} \pi x\right),
$$

where $0<a<1, b$ is a positive odd integer, and $a b>1+3 \pi / 2$. We know now that the graph of the Weierstrass function has Hausdorff dimension greater than one, which provides some explanation for this pathological function's dearth of differentiability: in particular, one can easily show that differentiable functions have graphs of Hausdorff dimension 1. It is also well known that that there exist continuous functions $f:[0,1] \rightarrow \mathbf{R}$ with graph of Hausdorff dimension 2 .

It turns out that the seemingly pathological behavior of a continuous function with a graph of large dimension is the rule rather than the exception. Balka, Darji and Elekes recently showed [1] that for any compact uncountable metric space $K$, within the space of continuous functions $f: K \rightarrow \mathbf{R}$, those with graphs of Hausdorff

https://doi.org/10.5186/aasfm.2021.4622

2020 Mathematics Subject Classification: Primary 28A78.

Key words: Hausdorff dimension, Lebesgue measure, injective projections, union of lines, union of disjoint planes.

The third author was supported by the Hungarian National Research, Development ad Innovation Office - NKFIH, 124749 and 129335. 
dimension $\operatorname{dim} K+1$ are prevalent in a measure-theoretic sense. (In this paper dim always denotes Hausdorff dimension.) Intuition might suggest that these graphs rely heavily on local oscillations to increase their Hausdorff dimension, and therefore would not be injective. Many of the classical constructions take advantage of this strategy. For example, the Weierstrass function fails to be injective in the most spectacular way: it lacks monotonicity on all arbitrarily short intervals. This is an example of a continuous non-injective map with a large graph. More recently, Eiderman and Larsen found that it is possible to trade continuity for injectivity: they constructed [4] an injective non-continuous function on $[0,1]$ whose graph has Hausdorff dimension 2.

It is therefore natural to ask whether there exist injective and continuous realvalued functions that have large graph dimension. Such a function cannot rely on local oscillations in the same way as the Weierstrass function: clearly, if a continuous real-valued injective function is defined on an interval, then it is monotone and necessarily has dimension one. Hence, such a function must be defined on some carefully chosen set.

In the present paper, we answer this question in the affirmative. We construct compact sets $K_{1}, K_{2} \subset[0,1]$ of dimension $t$, as well as a homeomorphism $f: K_{1} \rightarrow K_{2}$ so that $\operatorname{dim} \operatorname{graph}(f)=1+t$, for any desired value of $t \in[0,1]$. This dimension is maximal because $\Gamma$ is contained in the Cartesian product $K_{1} \times[0,1]$. The construction of such a function reduces to assembling a set $\Gamma \subset[0,1]^{2}$ which projects injectively onto $K_{1}$ in the domain and $K_{2}$ in the codomain. Our method of assembling $\Gamma$ is a modified Venetian blind construction, in which we make extra effort to ensure injectivity of the projections. This generalizes in many ways: first, the two coordinate axes can be replaced with any pair of (not necessarily orthogonal) lines, and this pair of lines can in turn be replaced with any finite or countable collection of lines. It is also natural to consider projections into lines inside the ambient space $\mathbf{R}^{d}$ rather than $\mathbf{R}^{2}$. This is our main result.

Theorem 1.1. Let $\mathscr{L}$ be a finite or countable set of lines in $\mathbf{R}^{d}$. Then for any $t \in[0,1]$, there exists a compact set $\Gamma \subseteq[0,1]^{d}$ with $\operatorname{dim}_{H} \Gamma=d-1+t$, such that each orthogonal projection $\pi_{\ell}: \Gamma \rightarrow \ell$ is injective with $\operatorname{dim} \pi_{\ell}(\Gamma)=t$. Furthermore, consider each of the following statements:

(1) The set $\Gamma$ has positive $(d-1+t)$-capacity and infinite $(d-1+t)$-dimensional Hausdorff measure.

(2) The $t$-dimensional Hausdorff measure of every $\pi_{\ell}(\Gamma)$ is 0 .

If $t=0$, then (1) holds; if $t=1$, then (2) holds; and if $t \in(0,1)$, then one can choose either of (1) or (2) to hold.

In $\mathbf{R}^{d}$ we can consider projections into linear subspaces $w$ of any dimension. Analogously, we construct large $\Gamma$ such that the projection $\pi_{w}: \Gamma \rightarrow w$ is injective and has dimension $\operatorname{dim} \pi_{w}(\Gamma)=\operatorname{dim} w-s$ for any prescribed $s \in[0,1]$. In this most generalized form, we once again find an easy upper bound on $\operatorname{dim} \Gamma$ : since $\Gamma$ is contained in an isometric image of $\pi_{w}(\Gamma) \times w^{\perp}$, we have $\operatorname{dim} \Gamma \leq d-s$. This maximum possible dimension is precisely the one that we obtain as our first corollary.

Corollary 1.2. Fix $d \geq 2$, and let $\mathscr{W}$ be a finite or countable collection of linear subspaces in $\mathbf{R}^{d}$ (not necessarily all of the same dimension). Then for any $s \in[0,1]$ there exists a compact set $\Gamma \subseteq[0,1]^{d}$ with $\operatorname{dim} \Gamma=d-s$, such that each projection $\pi_{w}: \Gamma \rightarrow w$ is injective with $\operatorname{dim} \pi_{w}(\Gamma)=\operatorname{dim} w-s$. 
Without the injectivity of the projections, this was proved in Claim 2.4 of [2]. Next, by applying Theorem 1.1 to the standard basis vectors, we obtain the following corollary on the existence of homeomorphisms whose graphs have large dimension. The correspondence between bijective (specifically, coordinate-wise injective) functions $f$ and sets $\Gamma$ injective onto each coordinate axis is clear. That $f$ is a homeomorphism follows easily from the compactness of the graph $\Gamma$.

Corollary 1.3. For any $d \geq 1$ and $t \in[0,1]$, there exist compact $K, K_{1}, \ldots, K_{d} \subset$ $[0,1]$ with dimension $t$ and a coordinate-wise injective homeomorphism $f: K \rightarrow$ $K_{1} \times \cdots \times K_{d}$ such that dimgraph $(f)=d+t$. Further, if $t>0$ then each of $K, K_{1}, \ldots K_{d}$ has $t$-dimensional Hausdorff measure 0.

Denoting by $A(d, k)$ the set of $k$-planes in $\mathbf{R}^{d}$, we can place a natural metric on $A(d, k)$ through association with $\mathbf{R}^{(k+1)(d-k)}$. Through this metric one can investigate the relationship between the Hausdorff dimension of a collection $E \subset A(d, k)$ and the size (Lebesgue measure or dimension) of its union $B:=B_{E}$ in $\mathbf{R}^{d}$. In [8, Theorem 1.3] Oberlin shows that if $B$ has Lebesgue measure zero then $\operatorname{dim} E \leq(k+1)(d-$ $k)-k$, and provides examples which demonstrate that this is tight. Concerning the Hausdorff dimension of $B$, in [5, Corollary 1.12] Héra proves that $\operatorname{dim} B \geq$ $k+\operatorname{dim} E /(k+1)$, and provides examples which are tight in some specific cases. More concretely, for any $s \in[0,(k+1)(d-k)]$ she constructs a collection of $k$-planes $E \subset A(d, k)$ with $\operatorname{dim} E=s$ such that the union of the $k$-planes has the following Hausdorff dimension,

$$
h(k, s):= \begin{cases}s-k\left\lceil\frac{s}{k+1}\right\rceil+2 k & \text { if }\left\lceil\frac{s}{k+1}\right\rceil \geq \frac{k+s}{k+1}, \\ k+\left\lceil\frac{s}{k+1}\right\rceil & \text { if }\left\lceil\frac{s}{k+1}\right\rceil \leq \frac{k+s}{k+1}\end{cases}
$$

Héra also formulates the conjecture that this is the best construction in the sense that whenever $E \subset A(d, k)$ with $\operatorname{dim} E=s$ and $B$ is the the union of the $k$-planes of $E$ then $\operatorname{dim} B \geq h(k, s)$.

The examples furnished by Héra and Oberlin involve collections of $k$-planes which may intersect one another or are parallel. Since the objective is minimizing the size of $B$, it is not clear whether these intersections or collections of parallel $k$-planes are an important component of the construction. As an application of Corollary 1.3, we present constructions corresponding to those in [8] and [5], with the additional property that they consist of disjoint, nonparallel $k$-planes. We found in Theorem 1.1 that requiring injectivity of a continuous function will not necessarily reduce the Hausdorff dimension of its graph; here we find an analogous statement, that requiring $k$-planes to be disjoint and non-parallel does not necessarily increase the size of their union.

Theorem 1.4. Let $d, k \in \mathbf{N}$ with $d \geq k+2$.

(i) There exists a compact set of disjoint, nonparallel $k$-planes $E \subset A(d, k)$ with $\operatorname{dim} E=(k+1)(d-k)-k$ so that $B$, the union of $k$-planes in $E$, has Lebesgue measure zero.

(ii) For any $s$ which satisfies $0 \leq s \leq \operatorname{dim} A(d, k)=(k+1)(d-k)$, there exists a compact set of disjoint non-parallel $k$-planes $E \subset A(d, k)$ with $\operatorname{dim} E=s$ such that $B$, the union of $k$-planes in $E$, has Hausdorff dimension $\operatorname{dim} B \leq h(k, s)$ for the function $h(k, s)$ defined in (1.1).

Note that since any compact set $E$ has a compact subset of any given dimension less than $\operatorname{dim} E$ we can also get $E$ with smaller than the above prescribed dimension. 
This observation, in combination with (i) and the result of [8] that if $B$ has Lebesgue measure zero then $\operatorname{dim} E \leq(k+1)(d-k)-k$, gives the immediate corollary that we may exchange any such collection $E$ for another consisting of disjoint, nonparallel planes.

Corollary 1.5. Suppose $E \subset A(d, k)$ such that $B$, the union of those $k$-planes in $E$, has Lebesgue measure zero. Then there exists a compact set $E^{\prime} \subset A(d, k)$ consisting of disjoint, nonparallel $k$-planes such that $\operatorname{dim} E^{\prime}=\operatorname{dim} E$, with the property that $B^{\prime}$, the union of the $k$-planes in $E^{\prime}$, has Lebesgue measure zero.

We now consider one final application of Theorem 1.1. It is well known that, for a collection of nonvertical lines in the plane which covers a vertical line, the union must have Hausdorff dimension 2. In fact, this is essentially the same as the classical result of Davies [3] which states that every Besicovitch set in the plane must have Hausdorff dimension 2. One can ask what we can say in the opposite situation: if a collection of lines in the plane intersects a vertical line $w$ in a small set, does this imply that the union of the lines is small? The answer is clearly in the negative: for example, taking all non-vertical lines through a fixed point of $w$ is a counter-example. There are two natural ways to exclude this triviality: we could request the chosen lines to intersect $w$ in distinct points; or alternatively, we can require small intersections not only with $w$ but with more than one vertical line. By combining Theorem 1.1 with duality and projection theorems we show that even if we have both requirements it is possible that the intersection with the prescribed vertical lines are very small despite the union of the lines being very large. In fact, more generally we can construct a collection of hyperplanes in $\mathbf{R}^{d}$ with these properties.

Theorem 1.6. Let $d \geq 2$ and let $w_{1}, w_{2}, \ldots$ be a countable collection of parallel lines in $\mathbf{R}^{d}$. Then there exists a compact collection $H$ of hyperplanes in $\mathbf{R}^{d}$, not parallel to the lines $w_{i}$, such that every point of every $w_{i}$ intersects at most one $h \in H$, the set $F=\bigcup_{h \in H} h$ has positive Lebesgue measure, and $\operatorname{dim}\left(F \cap w_{i}\right)=0$ for every $w_{i}$.

Our paper is organized as follows. In Section 2, we deduce Corollary 1.2 and Theorem 1.6 from Theorem 1.1. In Section 3 we prove Theorem 1.4, using as a crucial ingredient the homeomorphisms furnished by Corollary 1.3. In Section 4 we construct a suitable set $\Gamma$ towards proving Theorem 1.1. There we also prove various geometric lemmas relating to our construction. Finally, in Section 5 we verify that $\Gamma$ and its projections have the alleged dimensions.

\section{Proofs of the direct applications of our main result}

\subsection{Generalization to higher dimensional subspaces.}

Proof of Corollary 1.2. Let $\mathscr{L}$ be a collection of lines such that for each $w \in \mathscr{W}$ there is some $\ell_{w} \in \mathscr{L}$ such that $\ell_{w} \subset w$. By Theorem 1.1, there exists a compact set $\Gamma$ of Hausdorff dimension $d-s$ such that $\operatorname{dim}_{\operatorname{proj}_{\ell}}=1-s$ for every $\ell \in \mathscr{L}$. Since the projections $\pi_{\ell}: \Gamma \rightarrow \ell$ are injective, so are the projections $\pi_{w}: \Gamma \rightarrow w$. Hence, it suffices to show that $\operatorname{dim} \pi_{w}(\Gamma)=\operatorname{dim} w-s$. Because $\Gamma$ is contained in some isometric image of $w^{\perp} \times \pi_{w}(\Gamma)$, we have $\operatorname{dim} \Gamma \leq d-\operatorname{dim} w+\operatorname{dim} \pi_{w}(\Gamma)$, which implies $\operatorname{dim} \pi_{\omega}(\Gamma) \geq \operatorname{dim} w-s$. As for the upper bound, by the inclusion $\ell_{w} \subset w$ we have that $\pi_{w}(\Gamma)$ is contained in some isometric image of $\left(w \cap \ell_{w}^{\perp}\right) \times \pi_{\ell_{w}}(\Gamma)$, which has dimension $\operatorname{dim} w-s$. 


\subsection{Large union of hyperplanes with small injective sections.}

Proof of Theorem 1.6. For any $x \in \mathbf{R}^{d-1}$ let $v_{x}$ denote the "vertical" line $\{x\} \times \mathbf{R}$ in $\mathbf{R}^{d}$. Without loss of generality we can suppose that the parallel lines $w_{i}$ are vertical; that is, they are of the form $w_{i}=v_{x_{i}}$ for some $x_{i} \in \mathbf{R}^{d-1}$. For any $(a, b) \in \mathbf{R}^{d-1} \times \mathbf{R}$ let $P_{a, b}$ denote the hyperplane $\left\{(x, y) \in \mathbf{R}^{d-1} \times \mathbf{R}: y=a \cdot x+b\right\}$ in $\mathbf{R}^{d}$, and for any $A \subset \mathbf{R}^{d}$ let $E(A):=\bigcup_{(a, b) \in A} P_{a, b}$. Then, we have

$$
E(A) \cap v_{x}=\left\{(x, a \cdot x+b) \in \mathbf{R}^{d-1} \times \mathbf{R}:(a, b) \in A\right\} \quad\left(x \in \mathbf{R}^{d-1}\right),
$$

and therefore the map $A \mapsto E(A) \cap v_{x}$ is a scaled copy of the orthogonal projection of $A$ to a line in the direction $(x, 1)$.

For each $i$ we let $\ell_{i}$ be a line in $\mathbf{R}^{d}$ with direction $\left(x_{i}, 1\right)$ and apply Theorem 1.1 to this collection with $t=0$. This yields a compact set $\Gamma \subset \mathbf{R}^{d}$ of positive $(d-$ 1)-capacity such that $\left.\pi_{\ell_{i}}\right|_{\Gamma}$ is injective with $\operatorname{dim} \pi_{\ell_{i}}(\Gamma)=0$. Now we take $H:=$ $\left\{P_{a, b}:(a, b) \in \Gamma\right\}$ and $F:=\bigcup_{h \in H} h$. Then $H$ is a compact collection of $(d-1)$ dimensional hyperplanes in $\mathbf{R}^{d}$, not parallel to the lines $w_{i}$, and also $F=E(\Gamma)$. The projection of $\Gamma$ into the line $\ell_{i}$ in the direction $\left(x_{i}, 1\right)$ corresponds to the intersection $F \cap w_{i}$. Since these projections are injective, every point of each $w_{i}$ is contained in at most one $h \in H$. It is also clear that $\operatorname{dim}\left(F \cap w_{i}\right)=\operatorname{dim} \pi_{\ell_{i}}(\Gamma)=0$ for every $w_{i}$.

It remains to check that $F=\bigcup_{h \in H} h$ has positive Lebesgue measure. By a result of Mattila [7, Corollary 9.10], if a set has positive $m$-capacity then its projection to almost every $m$-dimensional subspace has positive Lebesgue measure. We can apply this with $m=1$ and deduce that the projection of $\Gamma$ to almost every line through the origin has positive Lebesgue measure. Thus almost every vertical slice $v_{x} \cap F$ has positive measure, so by Fubini, $F$ has positive Lebesgue measure.

\section{Disjoint non-parallel $k$-planes}

In this section we prove Theorem 1.4, which consists of modifications of constructions given in [5] and [8]. In both cases we present constructions with the same Hausdorff dimension as those previously presented, with the additional property that the $k$-planes used are disjoint and non-parallel (whereas in [5] and [8] they were not).

As stated in the introduction, $A(d, k)$ denotes the set of $k$-dimensional affine subspaces in $\mathbf{R}^{d}$. We use a matrix formulation of the encoding of $A(d, k)$ used in [8]. Given a pair $\left(Y, y_{0}\right)$, where $Y$ is a $(d-k) \times k$ matrix and $y_{0}$ is a $(d-k) \times 1$ vector, we define the following $k$-plane,

$$
P\left(Y, y_{0}\right):=\left\{\left(x, y_{0}+Y \cdot x\right): x \in \mathbf{R}^{k}\right\} .
$$

Note that this encoding cannot represent all $k$-planes: if a $k$-plane does not pass through a point where the first $k$ coordinates are 0 , then it cannot be encoded in this form. For example, in $\mathbf{R}^{2}$, lines parallel to the $y$ axis cannot be written as $y=m x+b$. However, since this restriction is very weak, almost every plane in $A(d, k)$ can be represented in this way and this is sufficient for our considerations. Having encoded almost all elements of $A(d, k)$ as points in $\mathbf{R}^{(k+1)(d-k)}$, we inherit a metric on these $k$-planes from the Euclidean metric on $\mathbf{R}^{(k+1)(d-k)}$.

The proofs of the two parts of Theorem 1.4 are similarly structured. They seek to create a collection of disjoint, nonparallel $k$-planes $E$ so that $E$ is large, yet the union of those planes found in $E$ is small. This is accomplished by utilizing the function $f$ furnished by Corollary 1.3. In Equation (3.1) one may interpret $Y$ as the orientation and $y_{0}$ the displacement of the given $k$-plane. In our proof we will determine $Y$ and 
the first $(d-k)-1$ coordinates of $y_{0}$ by applying $f$ to the $(d-k)$ th coordinate of $y_{0}$. The large dimension of the graph of $f$ will provide the largeness of $E$ while the injectivitiy of $f$ will ensure that such a family of lines is not parallel.

\section{1. $B$ has Lebesgue measure zero.}

Proof of Theorem 1.4, part (i). Let $\lambda_{d}$ denote the $d$-dimensional Lebesgue measure. By Corollary 1.3, there exists a compact set $K \subset[0,1]$ with dimension 1 and Lebesgue measure 0 , as well as a continuous entry-wise injective function $f: K \rightarrow \mathbf{R}^{(k+1)(d-1-k)}$ such that dim graph $(f)=(k+1)(d-1-k)+1$. We view the codomain $\mathbf{R}^{(k+1)(d-1-k)}$ as the space of pairs of $(d-1-k) \times k$ and $(d-1-k) \times 1$ matrices over $\mathbf{R}$, by splitting $f$ into $f_{1}: K \rightarrow \mathbf{R}^{(d-1-k) \times k}$ and $f_{2}: K \rightarrow \mathbf{R}^{(d-1-k) \times 1}$. Then we define the following collection of $k$-planes.

$$
E:=\left\{P\left(Y, y_{0}\right): Y=\left[\frac{f_{1}(t)}{0 \cdots 0}\right], y_{0}=\left[\frac{f_{2}(t)}{t}\right], y_{0}^{d-k}=t \in K\right\}
$$

where $P\left(Y, y_{0}\right)$ is defined in $(3.1)$.

The function $f(t)$ determines the orientation and positioning of a single $k$-plane lying in $\mathbf{R}^{d-1} \times\{t\}$ for a given $t \in K$. Then $B \subset \mathbf{R}^{d-1} \times K$, and therefore this set satisfies $\lambda_{d}(B) \leq \lambda_{d}\left(\mathbf{R}^{d-1} \times K\right)=0$, where the last equality is furnished by $\lambda_{1}(K)=0$. Furthermore, our representation of $E \subset A(d, k)$ is simply graph $(f) \times\{0\} \subset A(n, k)$, viewing elements of $A(n, k)$ by their identification in $\mathbf{R}^{(k+1)(d-k)}$. Then $\operatorname{dim} E=$ $\operatorname{dim} \operatorname{graph}(f)=(k+1)(d-k-1)+1=(k+1)(d-k)-k$, as needed. Additionally, each $k$-plane in $E$ is disjoint since each $k$-plane is contained within a different slice $\mathbf{R}^{d-1} \times\{t\}$. Since $f$ is injective in each coordinate, each of the $k$-planes will have a different value for $Y_{1,1}$ in particular. Since this coordinate is one component of the orientation of the $k$-planes, they will be nonparallel.

\section{2. $B$ has limited Hausdorff dimension.}

Proof of Theorem 1.4, part (ii). We modify the construction given in [5] to select only $k$-planes which are disjoint and nonparallel. Set $m=\lceil s /(k+1)\rceil$. If $m=0$ then $s=0$ and setting $E$ to a single $k$-plane suffices. If $m=1 \geq(k+s) /(k+1)$, then $s \leq 1$ and so by [6] taking $E$ any $s$-dimensional collection of disjoint, nonparallel $k$-planes produces $\operatorname{dim} B=k+s$.

If $m \geq(k+s) /(k+1)$ and $m \geq 2$, then using Corollary 1.3 we choose some $A \subset[0,1]$ with $\operatorname{dim} A=s-(k+1)(m-1) \in(0,1]$, as well as a coordinate-wise injective homeomorphism $f: A \rightarrow \mathbf{R}^{(k+1)(m-1)}$ with $\operatorname{dim} \operatorname{graph}(f)=(k+1)(m-1)+\operatorname{dim} A=s$. Once again we view the codomain $\mathbf{R}^{(k+1)(m-1)}$ as the space of pairs of $(m-1) \times k$ and $(m-1) \times 1$ matrices over $\mathbf{R}$, by splitting $f$ into two maps $f_{1}: K \rightarrow \mathbf{R}^{(m-1) \times k}$ and $f_{2}: K \rightarrow \mathbf{R}^{(m-1) \times 1}$. Then we define the following collection of $k$-planes,

$$
E:=\left\{P\left(Y, y_{0}\right): Y=\left[\begin{array}{c}
f_{1}(t) \\
\\
\hline 0 \cdots 0 \\
\hline 0 \cdots 0 \\
\vdots \cdot \vdots \\
0 \cdots 0
\end{array}\right], y_{0}=\left[\begin{array}{c}
f_{2}(t) \\
\frac{}{t} \\
\hline 0 \\
\vdots \\
0
\end{array}\right], y_{0}^{m}=t \in A\right\} .
$$


In this case, viewing elements of $A(n, k)$ by their identification in $\mathbf{R}^{(k+1)(d-k)}$, we have $E=\operatorname{graph}(f) \times\{0\} \subset A(d, k)$, which implies $\operatorname{dim} E=\operatorname{dim} \operatorname{graph}(f)=s$, as needed. Further, since $B$ is contained within $\mathbf{R}^{m+k-1} \times A$, we also have that $\operatorname{dim} B \leq m+k-1+\operatorname{dim} A=s-k\lceil s /(k+1)\rceil+2 k$. The $k$-planes are disjoint because, as before, they each lie in a different copy of $\mathbf{R}^{m+k-1}$, and they are nonparallel because $f_{1}$ is coordinate-wise injective.

Finally, if $m \leq(k+s) /(k+1)$, we again use Corollary 1.3 to choose some $A \subset[0,1]$ with $\operatorname{dim} A=0$, as well as a coordinate-wise injective homeomorphism $f: A \rightarrow \mathbf{R}^{(k+1) m}$ with $\operatorname{dim} \operatorname{graph}(f)=(k+1) m$. Then setting $E^{\prime}$ as we defined $E$ in equation (3.2) above (replacing $m$ with $m+1$ in the definition of $E^{\prime}$ ), we have $\operatorname{dim} E^{\prime}=(k+1) m \geq s$, while $B$ is contained within $\mathbf{R}^{m+k} \times A$. This implies $\operatorname{dim} B \leq m+k+\operatorname{dim} A=\lceil s /(k+1)\rceil+k$, as needed. Finally, since $E^{\prime}$ is closed we may take a compact $s$-dimensional subset $E$ of $E^{\prime}$ to complete the proof.

Remark 3.1. While both of these constructions are at least as strong as the best existing results, (i) is more complete than (ii) because, as it was mentioned in the introduction, there are still gaps in our understanding of the dimension case, regardless of whether the $k$-planes are required to be disjoint or nonparallel.

With some extra effort we can guarantee $\operatorname{dim} B=h(k, s)$ in Theorem 1.4 (ii) by augmenting $E$ with a suitably chosen simple collection of disjoint non-parallel $k$ planes; it is not difficult to increase $\operatorname{dim} B$ leaving $\operatorname{dim} E$ the same. However, this may not be interesting, since if one happens to get $\operatorname{dim} B<h(k, s)$ in Theorem 1.4 (ii) then this construction surpasses the current best known (even without the extra condition that the $k$-planes are disjoint and non-parallel). In fact, it would give a counter-example to the alread mentioned conjecture of Héra [5, Conjecture 1.16], which states that such example cannot exist. In other words, the conjecture of Héra would imply $\operatorname{dim} B=h(k, s)$ in Theorem 1.4 (ii).

On the other hand, in [8] it is shown that if $B$ has Lebesgue measure zero then $\operatorname{dim} E \leq(k+1)(d-k)-k$, and therefore (i) of Theorem 1.4 constructs an extremal example. This dichotomy explains why we have Corollary 1.5 for (i) and not (ii) of Theorem 1.4.

\section{The set $\Gamma$}

In this section, we construct $\Gamma$ and compute salient attributes of it that will affect dimension and measure computations in the following section.

4.1. Modification and extension of the collection of lines. As we will see later, it is prudent to replace our collection of lines $\mathscr{L}$ with a sequence satisfying a convenient collection of properties.

Lemma 4.1. Let $\mathscr{L}$ be a countable collection of lines in $\mathbf{R}^{d}$ which go through the origin. Then there exists a sequence $\ell_{1}, \ell_{2}, \ell_{3}, \ldots$ so that:

(1) Every $\ell \in \mathscr{L}$ appears in $\left\{\ell_{i}\right\}$ infinitely many times.

(2) Any $d$ consecutive lines in $\left\{\ell_{i}\right\}$ have linearly independent directions.

Proof. First, we take $H$ a $d$-1-dimensional subspace in $\mathbf{R}^{d}$ which does not contain any $\ell \in \mathscr{L}$, and let $e_{1}, \ldots e_{d-1}$ be lines in $H$ through the origin with linearly independent directions. Then enumerate the lines $\ell \in \mathscr{L}$ so that each appears infinitely often, and insert between each line the $d-1$ lines $e_{1}, \ldots, e_{d-1}$. This new enumeration satisfies our constraints. 
4.2. The construction of $\Gamma$. Here we construct a compact set $\Gamma \subseteq \mathbf{R}^{d}$ which, as we will argue in this section and the next, suffices to prove Theorem 1.1. It will depend on our choice of two sequences, $n_{k}$ and $a_{k}$, which we will specify in Lemma 4.4, but for now we define $\Gamma$ for arbitrary positive real sequences $n_{k}$ and $a_{k}$.

Definition 4.2. Let $\left(a_{k}\right)$ and $\left(n_{k}\right)$ be positive real sequences. For $h, j \in \mathbf{Z}$, we define the following interval on the line $\ell_{k}$ :

$$
I_{k}^{(h, j)}:=\left[h \cdot 2^{-n_{k}+a_{k}}+j \cdot 2^{-n_{k}+1}, h \cdot 2^{-n_{k}+a_{k}}+j \cdot 2^{-n_{k}+1}+2^{-n_{k}}\right],
$$

where by an interval $[a, b]$ on the line $\ell$ we mean the closed line segment connecting $a \cdot \hat{\ell}$ to $b \cdot \hat{\ell}$, where $\hat{\ell}$ is the unit vector in the direction of $\ell$. Observe that for fixed $k$, these are segments of the same length, and as we'll prove in Lemma 4.7 , these segments are disjoint for a suitable choice of $a_{k}$ and $n_{k}$.

For a line $\ell_{k}$, let $\pi_{\ell_{k}}: \mathbf{R}^{d} \rightarrow \ell_{k}$ be the orthogonal projection onto $\ell_{k}$. We define sets $\Gamma_{0} \supset \Gamma_{1} \supset \ldots$ by induction. We first set

$$
\Gamma_{0}=\Gamma_{1}=\cdots=\Gamma_{d}:=[0,1]^{d}
$$

Suppose that $\Gamma_{k-1}$ is the union of a collection $R_{k-1}$ of $2^{m_{k-1}}$ identical disjoint solid closed parallelotopes:

$$
\Gamma_{k-1}=\bigcup_{j=1}^{2^{m_{k-1}}} R_{k-1}^{(j)}
$$

where $m_{k-1}:=\log _{2}\left|R_{k-1}\right|$. Using these, we inductively define

$$
\Gamma_{k}:=\bigcup_{h \in \mathbf{Z}} \bigcup_{j=1}^{2^{m_{k-1}}} \begin{cases}\pi_{\ell_{k}}^{-1}\left(I_{k}^{(h, j)}\right) \cap R_{k-1}^{(j)} & \text { if this is a parallelotope } \\ \emptyset & \text { otherwise. }\end{cases}
$$

Finally, we define

$$
\Gamma:=\bigcap_{k \geq 1} \Gamma_{k}
$$

4.3. Interpreting $\Gamma$. We now motivate and illustrate this definition. We defined $\Gamma$ to be the intersection of a nested sequence of compact sets $\Gamma_{0} \supseteq \Gamma_{1} \supseteq \Gamma_{2} \supseteq \ldots$, where the $\Gamma_{k}$ are defined inductively in (4.2). Each $\Gamma_{k}$ is the disjoint union of $2^{m_{k}}$ identical closed parallelotopes $R_{k}^{\left(j^{\prime}\right)}$, for $j^{\prime}=1, \ldots, 2^{m_{k}}$; we will use $R_{k}$ to denote the collection of such $R_{k}^{\left(j^{\prime}\right)}$. We determined the size and relative positioning of these parallelotopes using positive real sequences $\left(n_{k}\right)$ and $\left(a_{k}\right)$, and in this section we will illustrate these geometric objects. In the next section we will estimate $m_{k}$ in terms of these sequences.

For the purposes of visualization consider the case when $a_{k}$ and $n_{k}$ are both rapidly increasing with $a_{k}<n_{k}$. When we assemble $\Gamma_{k}$ from $\Gamma_{k-1}$, from each parallelotope $R_{k-1}^{(j)}$ in $\Gamma_{k-1}$ we are taking many smaller parallelotopes $R_{k}^{\left(j^{\prime}\right)}$, as in Figure 1. Injective projection onto $\ell_{k}$ is a major desired feature of $\Gamma$. A natural way to guarantee such injectivity is to require the parallelotopes $R_{k}^{\left(j^{\prime}\right)}$ to be contained in preimages, under the projection $\pi_{\ell_{k}}: \mathbf{R}^{d} \rightarrow \ell_{k}$, of carefully chosen disjoint intervals in $\ell_{k}$. These intervals were first defined in (4.1) and are each of width $2^{-n_{k}}$. 


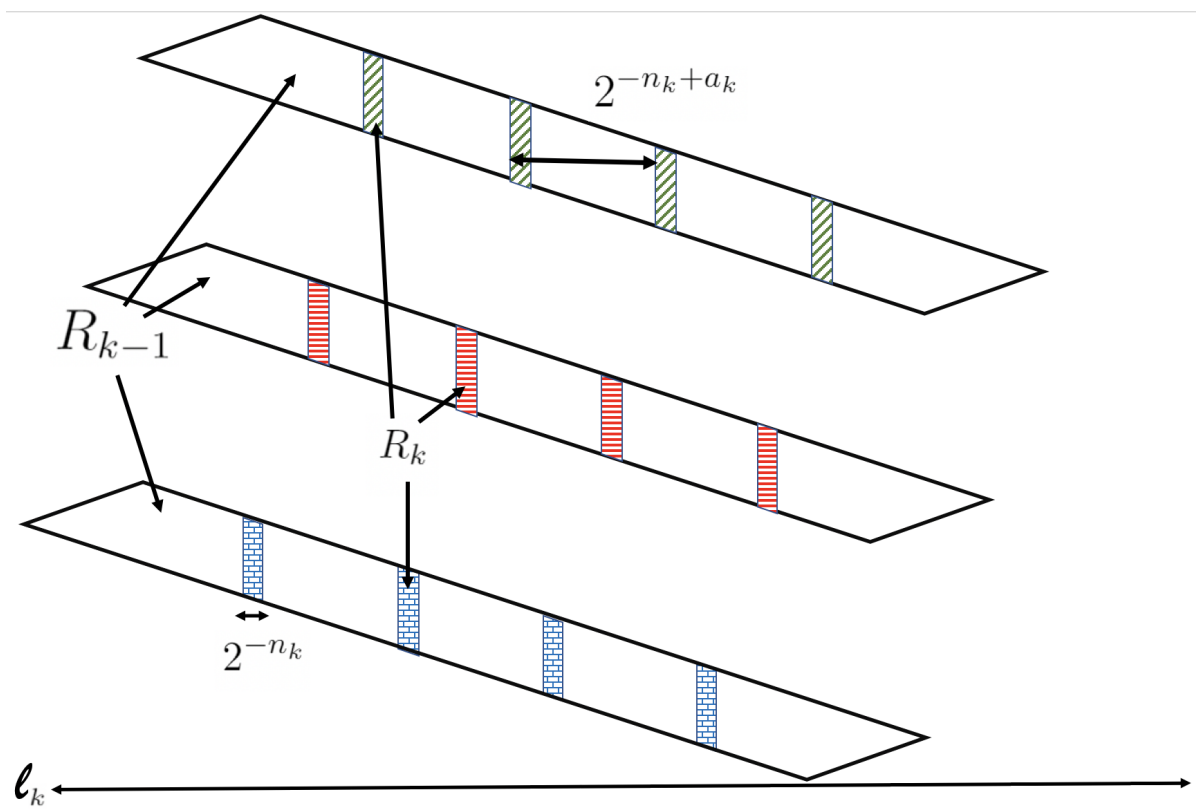

Figure 1. $R_{k}^{\left(j^{\prime}\right)}$ inside $R_{k-1}^{(j)}$ and associated distances.

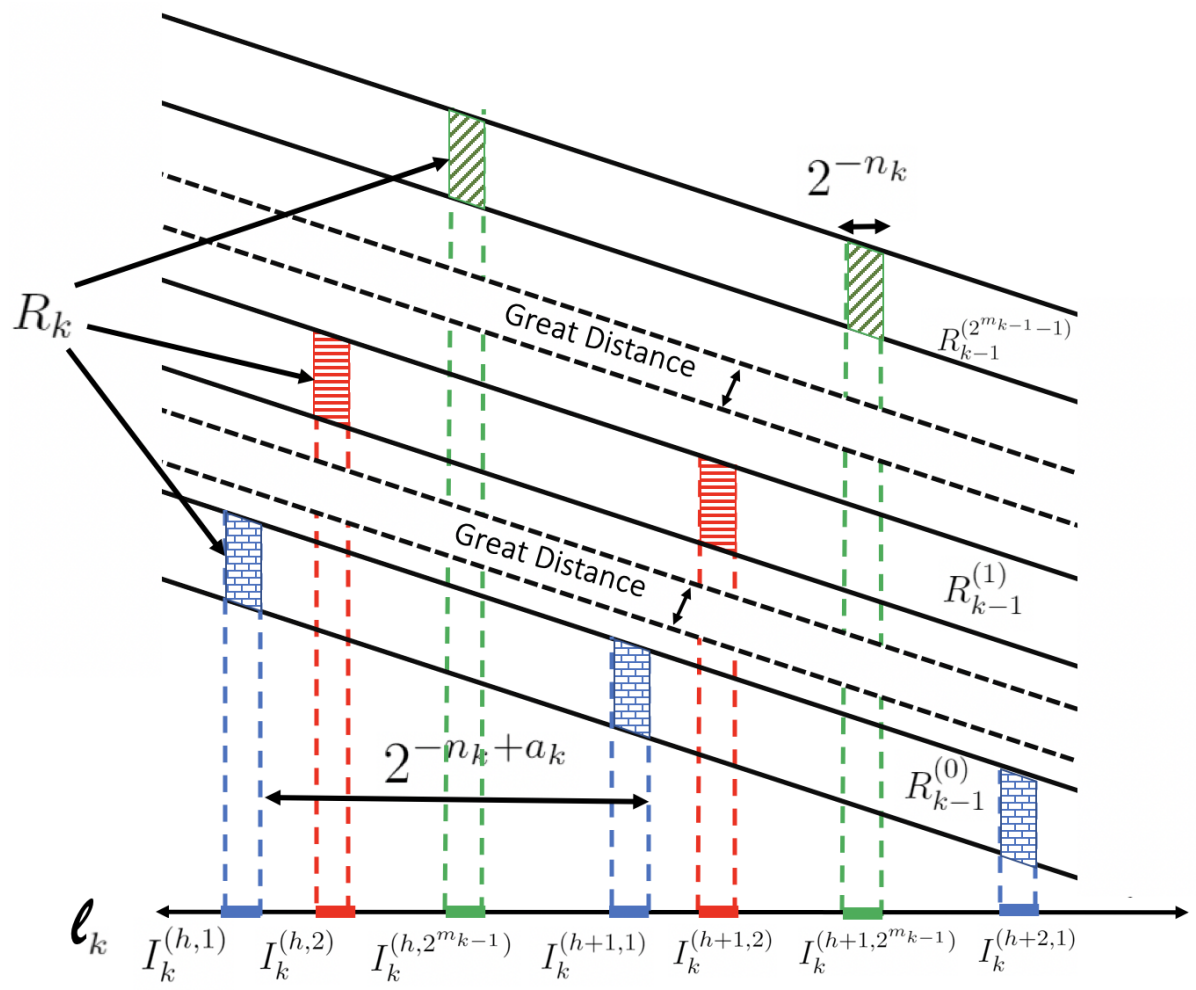

Figure 2. Illustration of the intervals defined in (4.1).

To motivate the choice of these intervals, we look ahead to our goal: to bound the Hausdorff dimension of $\Gamma$ from below. For this estimate, it will be necessary to place an a lower bound on the distance between two $R_{k}^{\left(j^{\prime}\right)}$ in $\Gamma_{k}$. If, inside a particular $R_{k-1}^{(j)}$, we place the new $R_{k}^{\left(j^{\prime}\right)}$ sufficiently close together, then the distance between the $R_{k-1}^{(j)}$ in $\Gamma_{k-1}$ will be very large compared to the distance between $R_{k}^{\left(j^{\prime}\right)}$ in $\Gamma_{k}$. This will ensure that the minimal distance between two parallelotopes in $\Gamma_{k}$ will be achieved only when the pair of polytopes originates from the same parallelotope $R_{k-1}^{(j)}$ 
in $\Gamma_{k-1}$. This is illustrated in Figure 2, where the distance between $R_{k}^{\left(j^{\prime}\right)}$ in different $R_{k-1}^{(j)}$ is much larger than the distance between those in the same $R_{k-1}^{(j)^{k}}$.

Our construction defined an offset of $2^{-n_{k}+a_{k}}$ from the start of one $R_{k}^{\left(j^{\prime}\right)}$ to the next. This is a large multiple of the width (measured in distance between opposite faces) of a single parallelotope $R_{k}^{\left(j^{\prime}\right)}$, so that the distance between two $R_{k}^{\left(j^{\prime}\right)}$ within the same $R_{k-1}^{(j)}$ is at least $2^{-n_{k}+a_{k}}-2^{-n_{k}}$.

Recall the definition of $I_{k}^{(h, j)}$ from Equation (4.1). For fixed $j$, the index $h$ determines an offset of $2^{-n_{k}+a_{k}}$. In Figure 2, these intervals are depicted as monochromatic. Next, we observe that the coefficient on $j$ is small relative to the coefficient on $h$. Hence, for a fixed $h$ we have that $j$ shifts the interval by a very small distance: in particular, twice the width of a single $R_{k}^{\left(j^{\prime}\right)}$. Our later constraints on the sequences $a_{k}$ and $n_{k}$ will imply that these intervals are disjoint as illustrated. Intervals of the same color in Figure 2 will be disjoint by definition, coming from the same $R_{k-1}^{(j)}$, and we will, in Lemma 4.4, force intervals of different colors to be disjoint by choosing $a_{k}$ large enough.

From a fixed $R_{k-1}^{(j)}$ we took as many parallelotopes as this separating distance will allow. The specification that we take only parallelotopes is necessary because it will happen that some $\pi_{\ell}^{-1}\left(I_{k}^{(h, j)}\right)$ intersects the parallelotope $R_{k-1}^{(j)}$ in one of its corners, or more generally any pair of adjacent sides, and in this case the intersection is not a true parallelotope. In Lemma 4.6, we show that such discarded sets are negligible so long as we take $n_{k}$ to grow sufficiently fast.

4.4. Estimating $\boldsymbol{m}_{\boldsymbol{k}}$. As is apparent from Definition 4.2 , the construction of $\Gamma$ is completely determined by the sequences $\left(n_{k}\right),\left(a_{k}\right)$, and $\left(\ell_{k}\right)$. In particular, in order to calculate the size of $\Gamma$ and its projections, we need good estimates on $m_{k}$ in terms of the given sequences $\left(n_{k}\right)$ and $\left(a_{k}\right)$.

Consider the projection of $R_{k-1}^{(j)}$ to $\ell_{k}$, and recall that in (4.2) we must discard those sets where the preimage of this projection is in a "corner" of $R_{k-1}^{(j)}$. In other words, we would like to estimate the length of the interval $I$ for which $\pi_{\ell_{k}}^{-1}\left(I_{k}^{(h, j)}\right) \cap$ $R_{k-1}^{(j)}$ is a parallelotope if and only if $I_{k}^{(h, j)} \subset I$. The following lemma gives the estimate we need.

Lemma 4.3. Let $\left(\ell_{k}\right)$ be the sequence of lines given by Lemma 4.1, let $\left(a_{k}\right)$ and $\left(n_{k}\right)$ be positive real sequences, and let $\Gamma=\Gamma\left(\left(\ell_{k}\right),\left(a_{k}\right),\left(n_{k}\right)\right)$ be as in Definition 4.2. There exist real numbers $\alpha_{d+1}, \alpha_{d+2}, \ldots$ and $\beta_{1}, \beta_{2}, \ldots$ that depend only on the sequence $\ell_{1}, \ell_{2}, \ldots$ such that for any $k>d$, under the assumption

$$
n_{i} \geq n_{k-d}+\alpha_{k}+\beta_{i} \quad(i=k-d+1, \ldots, k-1),
$$

the following holds. For each $j=1, \ldots, 2^{m_{k-1}}$ there exists a nonempty interval $I=I(j, k)$ of length

$$
2^{-n_{k-d}-\alpha_{k}-1} \leq|I| \leq 2^{-n_{k-d}-\alpha_{k}}
$$

such that for every $h \in \mathbf{Z}$ the set $\pi_{\ell_{k}}^{-1}\left(I_{k}^{(h, j)}\right) \cap R_{k-1}^{(j)}$ is a parallelotope if and only if $I_{k}^{(h, j)} \subset I$.

Proof. Fix $k$ and $j$ and let $R=R_{k-1}^{(j)}$. Let $I$ be the set of those real numbers $t$ for which the hyperplane $\pi_{\ell_{k}}^{-1}(\{t\})$ is between two opposite faces of the parallelotope $R$. Note that then indeed $\pi_{\ell_{k}}^{-1}\left(I_{k}^{(h, j)}\right) \cap R$ is a parallelotope if and only if $I_{k}^{(h, j)} \subset I$. By definition $I$ is nonempty if $R$ has two opposite faces such that their orthogonal 
projections to $\ell_{k}$ are disjoint and clearly the length of $I$ is the distance between these projections.

Note that by construction $R$ has $d$ pairs of opposite faces $\left(F_{k-d}, F_{k-d}^{\prime}\right), \ldots$, $\left(F_{k-1}, F_{k-1}^{\prime}\right)$ such that for every $i=k-d, \ldots, k-1$ the faces $F_{i}$ and $F_{i}^{\prime}$ are perpendicular to $\ell_{i}$ and the distance between the hyperplanes containing $F_{i}$ and $F_{i}^{\prime}$ is $2^{-n_{i}}$.

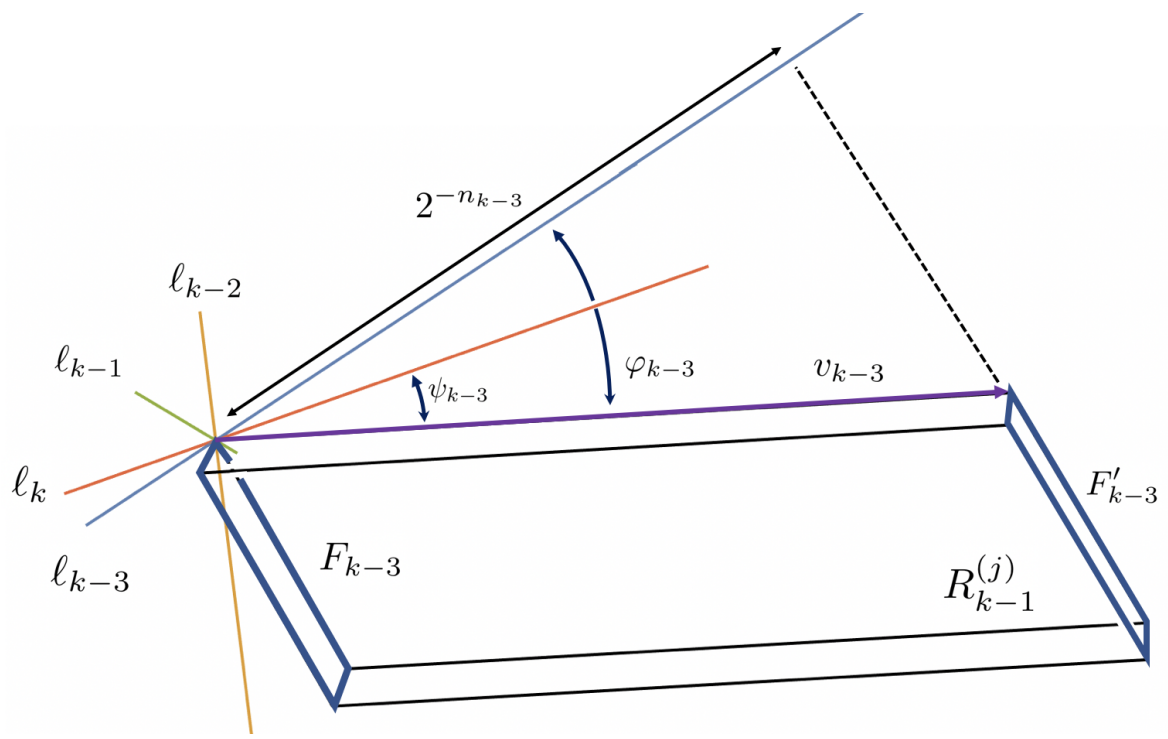

Figure 3. Illustration of the geometric ideas used in Lemma 4.3. In blue, yellow, and green we see the lines which define the shape of the parallelotope $R_{k-1}^{(j)}$. Each of these lines corresponds to a pair of faces. In the figure the faces corresponding to $\ell_{k-3}$ have been boldened and the vector $v_{k-3}$ and angle $\varphi_{k-3}$ drawn. The fourth line $\ell_{k}$ is the "target" onto which we will be projecting in the present step, so the angles $\psi$ inform the projection of $R_{k-1}^{(j)}$ onto this line.

For each $i=k-d, \ldots, k-1$ let $v_{i}$ be the vector such that $F_{i}+v_{i}=F_{i}^{\prime}$. Then $v_{i}$ is parallel to all faces of $R$ but $F_{i}$ and $F_{i}^{\prime}$, which implies that $v_{i}$ is perpendicular to every line $\ell_{k-d}, \ldots, \ell_{k-1}$ but $\ell_{i}$. Let $\varphi_{i}$ be the angle between $v_{i}$ and $\ell_{i}$ and let $\psi_{i}$ be the angle between $v_{i}$ and $\ell_{k}$.

Note that

$$
\left|v_{i}\right|=\frac{2^{-n_{i}}}{\cos \varphi_{i}}
$$

Since by (2) of Lemma 4.1 the directions of $\ell_{k-d}, \ldots, \ell_{k-1}$ are linearly independent, the directions of $v_{k-d}, \ldots, v_{k-1}$ are determined by $\ell_{k-d}, \ldots, \ell_{k-1}$, hence we obtain that the angles $\varphi_{i}$ depend only on the sequence of lines $\ell_{1}, \ell_{2}, \ldots$. We claim that $\varphi_{i} \neq \pi / 2$ for any $i=k-d, \ldots, k-1$ and $\psi_{k-d} \neq \pi / 2$. Indeed, $\varphi_{i}=\pi / 2$ would imply that $\ell_{k-d}, \ldots, \ell_{k-1}$ are all perpendicular to $v_{i}$ and $\psi_{k-d}=\pi / 2$ would imply that $\ell_{k-d+1}, \ldots, \ell_{k-1}, \ell_{k}$ are all perpendicular to $v_{k-d}$, which in both cases would contradict the linear independence assumption (2) of Lemma 4.1. The geometric setup is illustrated in Figure 3.

Since $|I|$ is the distance between $\pi_{\ell_{k}}\left(F_{k-d}\right)$ and $\pi_{\ell_{k}}\left(F_{k-d}^{\prime}\right)$, provided this distance is positive, we get that

$$
\left|\pi_{\ell_{k}}\left(v_{k-d}\right)\right|-\operatorname{diam}\left(\pi_{\ell_{k}}\left(F_{k-d}\right)\right) \leq|I| \leq\left|\pi_{\ell_{k}}\left(v_{k-d}\right)\right|,
$$


provided the lower estimate is positive. Define $\alpha_{k}$ and $\beta_{i}(i=k-d+1, \ldots, k-1)$ by

$$
2^{-\alpha_{k}}=\frac{\cos \psi_{k-d}}{\cos \varphi_{k-d}} \quad \text { and } \quad 2^{-\beta_{i}}=\frac{\cos \varphi_{i}}{2(d-1)}
$$

These numbers are well defined since none of these angles can be $\pi / 2$ and they depend only on the sequence of lines $\ell_{1}, \ell_{2}, \ldots$ and their own indices. Note that

$$
\left|\pi_{\ell_{k}}\left(v_{k-d}\right)\right|=\left|v_{k-d}\right| \cos \psi_{k-d}=\frac{2^{-n_{k-d}}}{\cos \varphi_{k-d}} \cos \psi_{k-d}=2^{-n_{k-d}-\alpha_{k}},
$$

which gives the upper estimate of (4.4) via (4.6). By this and (4.6), to get the lower estimate in (4.4) it is enough to show that

$$
\operatorname{diam}\left(\pi_{\ell_{k}}\left(F_{k-d}\right)\right) \leq 2^{-n_{k-d}-\alpha_{k}-1} .
$$

Using that projection cannot increase the distance, the edges of $F_{k-d}$ have lengths $\left|v_{k-d+1}\right|, \ldots,\left|v_{k-1}\right|$, and (4.5), we obtain

$$
\operatorname{diam}\left(\pi_{\ell_{k}}\left(F_{k-d}\right)\right) \leq \operatorname{diam}\left(F_{k-d}\right) \leq \sum_{i=k-d+1}^{k-1}\left|v_{i}\right|=\sum_{i=k-d+1}^{k-1} \frac{2^{-n_{i}}}{\cos \varphi_{i}} .
$$

On the other hand the assumption (4.3) and the definition of $\beta_{i}$ gives

$$
\frac{2^{-n_{i}}}{\cos \varphi_{i}} \leq \frac{2^{-n_{k-d}-\alpha_{k}-\beta_{i}}}{\cos \varphi_{i}}=\frac{2^{n_{k-d}-\alpha_{k}}}{2(d-1)}
$$

Combining these we get (4.8), which completes the proof.

The following lemma contains our requirements about the sequences $\left(a_{k}\right)$ and $\left(n_{k}\right)$ in the construction.

Lemma 4.4. Fix $t \in[0,1]$. Let $\left(\ell_{k}\right)$ be the sequence of lines given by Lemma 4.1, and let $\alpha_{d+1}, \alpha_{d+2}, \ldots$ and $\beta_{1}, \beta_{2}, \ldots$ be the sequences given by Lemma 4.3. There exist positive real sequences $\left(a_{k}\right)$ and $\left(n_{k}\right)$ such that for every $k>d$, all of the following conditions hold:

I. $n_{i} \geq n_{k-d}+\alpha_{k}+\beta_{i} \quad(i=k-d+1, \ldots, k-1)$.

II. $n_{k} \geq \beta_{k}+k$.

III. $n_{k}-a_{k} \geq n_{k-d}+\alpha_{k}+1$.

IV. $n_{k} \geq 2 k \cdot\left(2 n_{k-1}+a_{k-1}+n_{k-d}+\left|\alpha_{k}\right|+2\right)$.

V. $n_{k} \geq 4 k \cdot\left(2 n_{k-1}+a_{k-1}+n_{k-d}+\alpha_{k}+\alpha_{k+d}+3 d+2+\sum_{j=1}^{d-1}\left(n_{k-d+j}+\alpha_{k+j}\right)\right)$.

VI. $n_{k} \geq 4 k \cdot\left(\alpha_{k+d}+2\right)$.

VII. $a_{k} \geq 2 n_{k-1}-a_{k-1}+2$.

VIII. $n_{k} \geq 2 k^{2}$.

IX. $n_{k+1}-a_{k+1} \geq n_{k}$.

X. $\left(\log _{2} n_{k}\right) / n_{k} \leq 1 /(4 k)$.

XI. $\lim _{k \rightarrow \infty} a_{k} / n_{k}=1-t$.

XII. Consider the following statements:

(1) For large enough $k$, we have $1-t \geq \frac{a_{k}}{n_{k}}+\frac{1}{k}$.

(2) For large enough $k$, we have $1-t \leq \frac{a_{k}}{n_{k}}-\frac{1}{k}$.

If $t=0$, then (1) holds; if $t=1$, then (2) holds; and if $t \in(0,1)$, then we can choose either of (1) or (2) to hold. 
Remark 4.5. In Lemma 4.4, the choice of imposing growth condition (1) or (2) on $a_{k} / n_{k}$ corresponds to the choice of alternative (1) or (2) in Theorem 1.1. We will justify this correspondence in Section 5 .

Proof of Lemma 4.4. For every $k$, let us initially set

$$
\nu_{k}^{\prime}=1-t \pm \frac{1}{k}, \quad \nu_{k}=\left\{\begin{array}{ll}
\nu_{k}^{\prime} & \text { if } \nu_{k}^{\prime} \in(0,1), \\
1 / 2 & \text { otherwise }
\end{array} \quad \text { and } \quad a_{k}=\nu_{k} \cdot n_{k}\right.
$$

where we choose $+1 / k$ if we want (2) in property 4.4 and $-1 / k$ if we want (1) in property 4.4 . This choice satisfies properties 4.4 and 4.4 for any sequence $n_{k}$. We will now show that there is a choice of $n_{k}$ which increases rapidly enough to satisfy the remaining properties. Property 4.4 is satisfied for any sufficiently large $n_{k}$, since $\lim _{t \rightarrow \infty} t^{-1} \log _{2} t=0$, and similarly for property 4.4 .

By algebraically substituting every $a_{i}$ by $\nu_{i} \cdot n_{i}$ and using that $0<\nu_{i}<1$, we can see that all properties 4.4 through 4.4 are of the form $c \cdot n_{i} \geq f\left(n_{1}, \ldots, n_{i-1}\right)$, where the constant $c>0$ and the function $f$ depend only on $i, d$ and the sequence of lines $\ell_{1}, \ell_{2}, \ldots$; also, property 4.4 consists of finitely many inequalities of this form. Therefore by induction all of these properties can be satisfied.

Now that we can estimate the width of the valuable space inside the projection of $R_{k-1}^{(j)}$ to $\ell_{k}$, we estimate the number of intervals that can fit into the projection of a single $R_{k-1}^{(j)}$. This allows us to effectively estimate the quantity of new parallelotopes $R_{k}^{\left(j^{\prime}\right)}$ born from a single $R_{k-1}^{(j)}$, which in turn allows us to estimate the number of parallelotopes $R_{k}^{\left(j^{\prime}\right)}$ inside $\Gamma_{k}$.

Lemma 4.6. Fix $t \in[0,1]$. Let $\left(a_{k}\right)$ and $\left(n_{k}\right)$ be real sequences given by Lemma 4.4, let $\left(\ell_{k}\right)$ be the sequence of lines given by Lemma 4.1, and let $\left(m_{k}\right)$ be the corresponding real sequences provided in Definition 4.2. For $k>d$, let $\alpha_{k}$ be as in Lemma 4.4. Then, for some $\tilde{\alpha}_{k} \in\left[\alpha_{k}-1, \alpha_{k}+2\right]$, we have

$$
m_{k}=n_{k}-a_{k}+m_{k-1}-n_{k-d}-\tilde{\alpha}_{k} .
$$

In particular, there exist real sequences $\left(\delta_{k}\right)_{k \geq d+1},\left(\varepsilon_{k}\right)_{k \geq d+1}$, and $\left(\varepsilon_{k}^{\prime}\right)_{k \geq d+1}$ with $\left|\delta_{k}\right|,\left|\varepsilon_{k}\right|,\left|\varepsilon_{k}^{\prime}\right| \leq 1 /(2 k)$ such that the following hold for $k>d$ :

i) We have $m_{k}=\left(1+\delta_{k}\right) n_{k}-a_{k}$.

ii) We have $-m_{k+d}+\sum_{j=1}^{d}\left(n_{k+j}-a_{k+j}\right)+d=a_{k}+\varepsilon_{k} n_{k}$.

iii) We have $-m_{k+d-1}+\sum_{j=1}^{d-1}\left(n_{k+j}-a_{k+j}\right)+d=a_{k}+\left(-1+\varepsilon_{k}^{\prime}\right) n_{k}$.

Proof. Let $k>d$. We estimate $m_{k}$, which we recall is entirely determined by our inductive definition (4.2).

Fix $j \in\left\{1, \ldots, 2^{m_{k-1}}\right\}$ and let $r_{k-1}^{j}$ be the quantity of $R_{k}^{\left(j^{\prime}\right)}$ inside $R_{k-1}^{(j)}$. By Property 4.4 of Lemma 4.4, the assumption (4.3) of Lemma 4.3 holds, so the conclusion holds as well. Let $I$ be the interval Lemma 4.3 gives. By construction, we have

$$
\left\lfloor\frac{|I|}{2^{-n_{k}+a_{k}}}\right\rfloor \leq r_{k-1}^{j} \leq\left\lceil\frac{|I|}{2^{-n_{k}+a_{k}}}\right\rceil .
$$

Combining this with (4.4) of Lemma 4.3 we obtain

$$
\left\lfloor 2^{n_{k}-a_{k}-n_{k-d}-\alpha_{k}-1}\right\rfloor \leq r_{k-1}^{j} \leq\left\lceil 2^{n_{k}-a_{k}-n_{k-d}-\alpha_{k}}\right\rceil .
$$

Using (4.4) of Lemma 4.4 and the fact that $x / 2 \leq\lfloor x\rfloor$ and $\lceil x\rceil \leq 2 x$ for any $x \geq 1$, this gives

$$
2^{n_{k}-a_{k}-n_{k-d}-\alpha_{k}-2} \leq r_{k-1}^{j} \leq 2^{n_{k}-a_{k}-n_{k-d}-\alpha_{k}+1}
$$


Since by definition $2^{m_{k}}=\sum_{j=1}^{2^{m_{k-1}}} r_{k-1}^{j}$ this implies that

$$
m_{k-1}+n_{k}-a_{k}-n_{k-d}-\alpha_{k}-2 \leq m_{k} \leq m_{k-1}+n_{k}-a_{k}-n_{k-d}-\alpha_{k}+1,
$$

which completes the proof of the first paragraph of the lemma.

Now we verify i). If we define

$$
\delta_{k}:=\left(m_{k-1}-n_{k-d}-\tilde{\alpha}_{k}\right) / n_{k},
$$

then an elementary calculation verifies that $m_{k}=\left(1+\delta_{k}\right) n_{k}-a_{k}$, so it remains to show that $\left|\delta_{k}\right| \leq 1 /(2 k)$. We show this by induction on $k$. As (by Definition 4.2) $m_{d}=0$, the base case $\left|\delta_{d+1}\right| \leq 1 / 2(d+1)$ is equivalent to $n_{d+1} \geq 2(d+1) \cdot\left(n_{1}+\tilde{\alpha}_{d+1}\right)$, which is implied by property 4.4 in Lemma 4.4 for $k=d+1$. Now let $k \geq d+2$, and assume that $\left|\delta_{k-1}\right| \leq 1 / 2(k-1)$. By definition, $\left|\delta_{k}\right| \leq\left(m_{k-1}+n_{k-d}+\left|\tilde{\alpha}_{k}\right|\right) / n_{k}$. Using i) for $k-1, \tilde{\alpha}_{k} \in\left[\alpha_{k}-1, \alpha_{k}+2\right]$ and $\left|\delta_{k-1}\right|<1$, we obtain

$$
\left|\delta_{k}\right| \leq \frac{\left(1+\delta_{k-1}\right) n_{k-1}-a_{k-1}+n_{k-d}+\left|\alpha_{k}\right|+2}{n_{k}} \leq \frac{2 n_{k-1}-a_{k-1}+n_{k-d}+\left|\alpha_{k}\right|+2}{n_{k}}
$$

which is at most $1 /(2 k)$ by property 4.4 of Lemma 4.4 . This proves i).

Next we verify ii). Towards this, we define

$$
\varepsilon_{k}:=\frac{-m_{k-1}+n_{k-d}+\tilde{\alpha}_{k}+\tilde{\alpha}_{k+d}+d+\sum_{j=1}^{d-1}\left(n_{k-d+j}+\tilde{\alpha}_{k+j}\right)}{n_{k}} .
$$

Using telescopic sums, we can compute using (4.10) that ii) holds with this choice of $\varepsilon_{k}$, so it remains to show that $\left|\varepsilon_{k}\right| \leq 1 /(2 k)$. If, using i), we replace $m_{k-1}$ with $\left(1+\delta_{k-1}\right) n_{k-1}-a_{k-1}$ in (4.12), then take the modulus of each term and use that $\left|\tilde{\alpha}_{i}\right| \leq\left|\alpha_{i}\right|+2$ for $i>d$, then property 4.4 in Lemma 4.4 gives that $\left|\varepsilon_{k}\right| \leq 1 /(4 k) \leq$ $1 /(2 k)$.

Lastly we verify iii). Noting the similarity to ii), if we define

$$
\varepsilon_{k}^{\prime}:=\varepsilon_{k}-\frac{\tilde{\alpha}_{k+d}}{n_{k}}
$$

then, using (4.10) for $k+d$ instead of $k$, a straightforward calculation shows that iii) holds with this choice of $\varepsilon_{k}^{\prime}$. Note that in the previous paragraph we proved the stronger estimate $\left|\varepsilon_{k}\right| \leq 1 /(4 k)$. Therefore, $\left|\varepsilon_{k}^{\prime}\right| \leq 1 /(2 k)$ follows from the triangle inequality applied to (4.13), in conjunction with property 4.4 of Lemma 4.4.

\subsection{Injectivity of $\pi_{\ell_{k}}: \Gamma \rightarrow \ell_{k}$.}

Lemma 4.7. Let $\left(\ell_{k}\right)$ be the sequence of lines given by Lemma 4.1, let $\left(a_{k}\right)$ and $\left(n_{k}\right)$ be the real sequences given by Lemma 4.4, and let $\Gamma=\Gamma\left(\left(\ell_{k}\right),\left(a_{k}\right),\left(n_{k}\right)\right)$ be the corresponding set. Then, for fixed large enough $k$, the intervals $I_{k}^{(h, j)}$ defined in (4.1) are disjoint for all distinct pairs $(h, j)$ with $h \in \mathbf{Z}, 1 \leq j \leq 2^{m_{k-1}}$.

Proof. We consider two intervals $I_{k}^{(h, j)}$ and $I_{k}^{\left(h^{\prime}, j^{\prime}\right)}$. If $h<h^{\prime}$, then the distance between the left endpoint of $I_{k}^{\left(h^{\prime}, j^{\prime}\right)}$ and the right endpoint of $I_{k}^{(h, j)}$ is

$$
\left(h^{\prime}-h\right) 2^{-n_{k}+a_{k}}+\left(j^{\prime}-j\right) 2^{-n_{k}+1}-2^{-n_{k}} \geq 2^{-n_{k}+a_{k}}-2^{-n_{k}+1+m_{k-1}}-2^{-n_{k}} .
$$

Next, property 4.4 of Lemma 4.4, in conjunction with Lemma 4.6 i), imply that that $a_{k}>2+m_{k-1}$ for $k$ large enough that $\left|\delta_{k-1}\right|<1$. Therefore the above distance is at least

$$
2^{-n_{k}+2+m_{k-1}}-2^{-n_{k}+1+m_{k-1}}-2^{-n_{k}} \geq 2^{-n_{k}+1}-2^{-n_{k}}=2^{-n_{k}} .
$$


With a positive separating distance, the intervals are disjoint. On the other hand, if $h=h^{\prime}$ then we may assume $j^{\prime}>j$, so the distance between the left endpoint of $I_{k}^{\left(h^{\prime}, j^{\prime}\right)}$ and the right endpoint of $I_{k}^{(h, j)}$ is

$$
\left(j^{\prime}-j\right) 2^{-n_{k}+1}-2^{-n_{k}} \geq 2^{-n_{k}+1}-2^{-n_{k}}=2^{-n_{k}},
$$

hence the intervals are disjoint in this case as well.

Having proved that the intervals $I_{k}^{(h, j)}$ are disjoint, we may proceed to injectivity.

Lemma 4.8. The map $\pi_{\ell_{k}}: \Gamma \rightarrow \ell_{k}$ is injective.

Proof. Suppose we have two points $x, y \in \Gamma$ with $\pi_{\ell_{k}}(x)=\pi_{\ell_{k}}(y)$, and take the subsequence $\left\{\ell_{k_{i}}\right\}$ which is identically $\ell_{k}$ (here we use property 4.1 of Lemma 4.1 ). Then the point $\pi_{\ell_{k}}(x)=\pi_{\ell_{k}}(y)$ on $\ell_{k}$ is contained in a sequence of intervals $I_{k_{i}}^{\left(h_{i}, j_{i}\right)}$. Since we proved above that these intervals are disjoint for large enough $k_{i}$, the choice of $\left(h_{i}, j_{i}\right)$ is unique. Then we have $x, y \in \pi_{\ell_{k}}^{-1}\left(I_{k_{i}}^{\left(h_{i}, j_{i}\right)}\right) \cap R_{k-1}^{\left(j_{i}\right)}$ since (by construction of $\Gamma_{k_{i}}$ ) this is the only parallelotope whose image under $\pi_{\ell_{k}}$ is $I_{k_{i}}^{\left(h_{i}, j_{i}\right)}$. Finally we check that

$$
\lim _{k \rightarrow \infty} \operatorname{diam}\left(R_{k}^{j}\right)=0 .
$$

First, recognize that $\operatorname{diam}\left(R_{k}^{j}\right)$ is constant across $j$ by construction. Recall those vectors $v_{i}$ and angles $\varphi_{i}$ used in the proof of Lemma 4.3 and the equations (4.5) and (4.7) relating them to each other and the constants $\beta_{i}$. Then using property 4.4 of Lemma 4.4 and that $d \geq 2$ we have

$$
\operatorname{diam}\left(R_{k}^{j}\right) \leq \sum_{i=k-d+1}^{k}\left|v_{i}\right|=\sum_{i=k-d+1}^{k} \frac{2^{-n_{i}}}{\cos \varphi_{i}}=\sum_{i=k-d+1}^{k} \frac{2^{-n_{i}+\beta_{i}}}{2(d-1)} \leq d \frac{2^{-k}}{2(d-1)} \leq 2^{-k}
$$

which indeed tends to 0 as $k \rightarrow \infty$. Showing that the diameters of the paralleletopes tends to 0 is enough to finish the proof because then $x=y$, so $\pi_{\ell_{k}}$ is indeed injective.

\section{Dimension and measure computations for $\Gamma$}

Fix a line $\ell \in \mathscr{L}$. In this section we prove the three estimates $\operatorname{dim} \pi_{\ell}(\Gamma) \leq t$, $\operatorname{dim} \Gamma \leq d-1+\operatorname{dim} \pi_{\ell}(\Gamma)$, and $\operatorname{dim} \Gamma \geq d-1+t$ in Subsections 5.1, 5.3, and 5.4 through 5.6, respectively. Together these clearly imply the first paragraph of Theorem 1.1.

Additionally, we show in Subsections 5.5 and 5.6 that for $t \in[0,1)$, the set $\Gamma$ has positive $(d-1+t)$-capacity provided $a_{k} / n_{k}$ satisfies the following estimate for sufficiently large $k$,

$$
1-t=\lim _{i \rightarrow \infty} \frac{a_{i}}{n_{i}} \geq \frac{a_{k}}{n_{k}}+\frac{1}{k}
$$

this is option (1) in Theorem 1.1, as well as property 4.4 of Lemma 4.4. Separately, we will argue in Subsection 5.2 that for $t \in(0,1]$, the $t$-dimensional Hausdorff measure of $\pi_{\ell}(\Gamma)$ is zero provided that for sufficiently large $k$, the ratio $a_{k} / n_{k}$ satisfies the following inequality,

$$
1-t=\lim _{i \rightarrow \infty} \frac{a_{i}}{n_{i}} \leq \frac{a_{k}}{n_{k}}-\frac{1}{k},
$$

which is option (2) in Theorem 1.1, as well as property 4.4 of Lemma 4.4. Observe that these conditions are not compatible, hence for $t \in(0,1)$ we cannot guarantee both (1) and (2) in Theorem 1.1. 
5.1. The upper bound $\operatorname{dim} \pi_{\ell}(\Gamma) \leq t$. It suffices to construct a sequence of finite covers $\left\{U_{i}\right\}$ for $\pi_{\ell}(\Gamma)$ such that for every $\varepsilon, \varepsilon^{\prime}>0$, for sufficiently large $i$, we have

$$
\sum_{j}\left(\operatorname{diam} U_{i}^{(j)}\right)^{t+\varepsilon}<\varepsilon^{\prime}
$$

We examine the natural sequence of coverings generated by our construction. Namely, there exists a subsequence $\left\{\ell_{k_{i}}\right\}$ of $\left\{\ell_{k}\right\}$ which is identically $\ell$, and as defined previously, the projection of $R_{k_{i}}=\cup_{j} R_{k_{i}(j)}^{(j)}$ into $\ell$ consists of $2^{m_{k_{i}}}$ intervals of width $2^{-n_{k_{i}}}$. Accordingly, we define the cover $\left\{U_{i}^{(j)}: j=1, \ldots, 2^{m_{k_{i}}}\right\}$ to be the collection of these intervals.

It follows that the above sum is $2^{m_{k_{i}}-n_{k_{i}}(t+\varepsilon)}$. By Lemma 4.4 property 4.4 and Lemma 4.6 i) we see as $i \rightarrow \infty$,

$$
\lim _{i \rightarrow \infty}\left[m_{k_{i}}-n_{k_{i}}(t+\varepsilon)\right]=\lim _{i \rightarrow \infty}\left[\left(\lim _{j \rightarrow \infty} \frac{a_{k_{j}}}{n_{k_{j}}}\right)-\frac{a_{k_{i}}}{n_{k_{i}}}+\delta_{k_{i}}-\varepsilon\right] n_{k_{i}}=-\infty
$$

as needed, since $\delta_{k_{i}} \rightarrow 0$ and $n_{k_{i}} \rightarrow \infty$.

5.2. Option (2) in Theorem 1.1. Here we verify that for $t \in(0,1]$, if we assume (5.2), then we have $H^{t}\left(\pi_{\ell}(\Gamma)\right)=0$. Utilizing the same sequence of covers $U_{i}$ defined above, we compute that

$$
\sum_{j}\left(\operatorname{diam} U_{i}^{(j)}\right)^{t}=2^{m_{k_{i}}-n_{k_{i}} t}
$$

Applying Lemma 4.4 property 4.4 and Lemma 4.6 i) as above, as well as (5.2), we see that

$$
\lim _{i \rightarrow \infty} m_{k_{i}}-n_{k_{i}} t=\lim _{i \rightarrow \infty}\left[\left(\lim _{j \rightarrow \infty} \frac{a_{k_{j}}}{n_{k_{j}}}\right)-\frac{a_{k_{i}}}{n_{k_{i}}}+\delta_{k_{i}}\right] n_{k_{i}} \leq \lim _{i \rightarrow \infty}\left[-\frac{1}{k_{i}}+\delta_{k_{i}}\right] n_{k_{i}}=-\infty,
$$

since $\left|\delta_{k_{i}}\right| \leq 1 / 2 k_{i}$ by Lemma 4.6 , and because $n_{k_{i}} \geq 2 k_{i}^{2}$ by property 4.4 of Lemma 4.4. Hence the $t$-dimensional Hausdorff measure of $\pi_{\ell}(\Gamma)$ is 0 , provided (5.2) holds.

5.3. The upper bound $\operatorname{dim} \Gamma \leq d-1+\operatorname{dim} \pi_{\ell}(\Gamma)$. This follows from the observation that $\Gamma$ is contained in some isometric image of $\ell^{\perp} \times \pi_{\ell}(\Gamma)$.

5.4. The setup for the lower bound on the size of $\Gamma$. To complete the proof of Theorem 1.1 it remains to prove $\operatorname{dim} \Gamma \geq d-1+t$ and, in order to get option (1), to show that if $t \in[0,1)$ and $(5.1)$ holds then $\Gamma$ has positive $(d-1+t)$-capacity and infinite $(d-1+t)$-dimensional Hausdorff measure.

Towards this, we define a mass distribution on $\Gamma$ in the natural way, starting with unit mass for $\Gamma_{0}$, uniformly distributing the mass from each paralellepiped in $\Gamma_{k-1}$ into the smaller sub-parallelotopes in $\Gamma_{k}$, and letting $\mu$ be the limiting mass distribution. Let $Q$ be a ball of diameter $2^{-q}$. By the mass distribution principle (see for example [?, pp. 61]), to prove that $\operatorname{dim} \Gamma \geq d-1+t$ it would suffice to show $\mu(Q) \leq 2^{-q s}$ for every $s<d-1+t$. In option (1) we also need capacity estimates, so to make the argument more consistent for the two situations, instead of the mass distribution principle we will apply (for both options) the following slightly stronger standard result, which we prove for completeness. 
Lemma 5.1. If $s>0$ and $\mu$ is a finite Borel measure supported on a compact set $K$, and

$$
\mu(Q) \leq \frac{2^{-q s}}{q^{2}} \quad \text { for any ball of diameter } 2^{-q} \text { for large enough } q,
$$

then the s-capacity of $K$ is positive and $K$ has infinite $s$-dimensional Hausdorff dimension.

Proof. By the definition of $s$-capacity $C_{s}$ (see [7]) in order to show $C_{s}(K)>0$ it is enough prove that $I_{s}(\mu)<\infty$, where $I_{s}(\mu)=\iint|x-y|^{-s} d \mu(y) d \mu(x)$ is the $s$-energy of $\mu$. As in [7], the inner integral can be rewritten as

$$
\int|x-y|^{-s} d \mu(y)=s \int_{0}^{\infty} r^{-s-1} \mu(B(x, r)) d r
$$

where $B(x, r)$ denotes the ball centered at $x$ with radius $r$. Since $\mu$ is a finite measure, this shows that in order to prove that $I_{s}(\mu)$ is finite it is enough to prove that for some fixed $r_{0}$ and $C$ (not depending on $x$ ) we have

$$
\int_{0}^{r_{0}} r^{-s-1} \mu(B(x, r)) d r \leq C .
$$

Applying the assumption of the lemma for $q=-\log _{2}(2 r)$ and taking $r_{0}$ small enough, we get that $\mu(B(x, r)) \leq(2 r)^{s} /\left(\log _{2}(2 r)\right)^{2}$ for $0<r<r_{0}$, which implies that the above inequality indeed holds for some finite constant $C$, which does not depend on $x$.

Finally, by [7, Theorem 8.7 (1)], we have that if $K$ has positive $s$-capacity then it also has infinite $s$-dimensional Hausdorff dimension, as needed.

By the above lemma, it remains to show the following.

Claim 5.2. (i) If $t \in[0,1)$ and (5.1) holds then we have (5.3) for $s=d-1+t$. (ii) If $t \in(0,1]$ and we assume only $\lim a_{i} / n_{i}=1-t$, then (5.3) holds for every $s \in[d-1, d-1+t)$.

To prove this claim, we consider two cases which together cover all possible values of $q$ : namely, either $2^{-n_{k+1}+a_{k+1}} \leq 2^{-q}<2^{-n_{k}}$, or $2^{-n_{k}} \leq 2^{-q}<2^{-n_{k}+a_{k}}$ for some uniquely chosen index $k$. It is clear that these cover all possible cases, because property 4.4 of Lemma 4.4 implies that $-n_{k+1}+a_{k+1} \leq-n_{k}<-n_{k}+a_{k}$.

5.5. Case 1: $2^{-n_{k+1}+a_{k+1}} \leq 2^{-q}<2^{-n_{k}}$. Here, the diameter of $Q$ is greater than the length of the shortest translation vector between two $R_{k+1}^{(i)}$, but small enough that a translated copy fits inside the containing $R_{k}^{\left(j^{\prime}\right)}$. This is illustrated in Figure 4.

In this case, we first obtain the following basic estimate,

$$
\mu(Q) \leq \mu\left(R_{k+d}^{(i)}\right) \cdot \#\left\{R_{k+d}^{(i)}: R_{k+d}^{(i)} \cap Q \neq \emptyset\right\} .
$$

By our construction the mass of each $R_{k+d}^{(i)}$ is $2^{-m_{k+d}}$, and the second factor can be bounded as follows,

$$
\begin{aligned}
\#\left\{R_{k+d}^{(i)}: R_{k+d}^{(i)} \cap Q \neq \emptyset\right\} & \leq \prod_{j=1}^{d} \max _{i}\left(\#\left\{R_{k+j}^{\left(i^{\prime}\right)} \in R_{k+j-1}^{(i)}: R_{k+j}^{\left(i^{\prime}\right)} \cap Q \neq \emptyset\right\}\right) \\
& \leq \prod_{j=1}^{d}\left[\frac{2^{-q}}{2^{-n_{k+j}+a_{k+j}}}\right] \leq \prod_{j=1}^{d} \frac{2^{-q+1}}{2^{-n_{k+j}+a_{k+j}}},
\end{aligned}
$$


where the first estimate holds since $Q$ can intersect only one $R_{k}^{(i)}$ and the second estimate holds because the shortest translation vector between any two $R_{k+j}^{(i)}$ has length $2^{-n_{k+j}+a_{k+j}}$ by our construction and all such sets must intersect $Q$. The final estimate holds by the fact that $\lceil x\rceil \leq 2 x$ for any $x \geq 1$, which holds here by using the case hypothesis and that the sequence $n_{k}-a_{k}$ is non-decreasing by property 4.4 of Lemma 4.4.

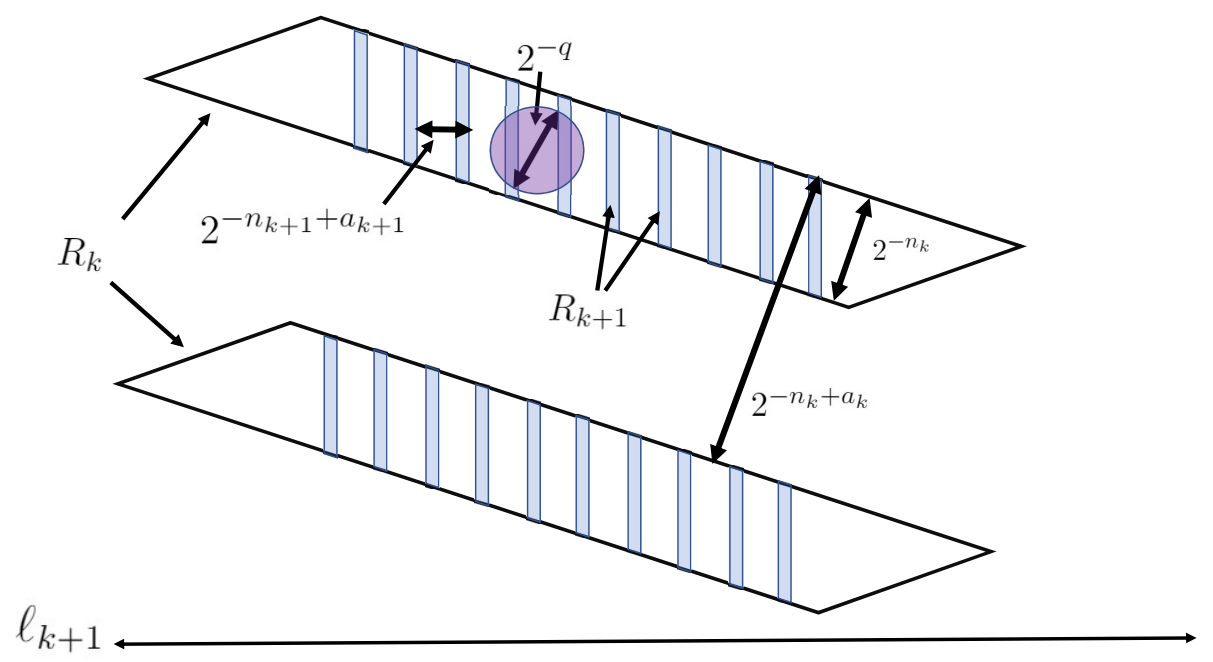

Figure 4. Positioning of $Q$ in Case 1.

Hence, (5.3) is implied by the following,

$$
-m_{k+d}+\sum_{j=1}^{d}\left(n_{k+j}-a_{k+j}\right)+d \leq q(d-s)-2 \log _{2} q .
$$

By Lemma 4.6 ii) this is equivalent to

$$
a_{k}+\varepsilon_{k} n_{k} \leq q(d-s)-2 \log _{2} q
$$

Because $d>s$ both in (i) and (ii), there exists $K_{1}$ so that for $q>K_{1}$ we have $q(d-s)-2 \log _{2} q$ is monotonically increasing in $q$. Since $n_{k}<q$ by the hypothesis of this case, we find that it is enough to prove

$$
s \leq d-\frac{a_{k}}{n_{k}}-\frac{2 \log _{2} n_{k}}{n_{k}}-\varepsilon_{k}
$$

To check (i) observe that if we assume (5.1) then (5.4) for $s=d-1+t$ is implied by $2 \log _{2} n_{k} / n_{k}+\varepsilon_{k} \leq 1 / k$, and this last inequality holds by property 4.4 , in conjunction with the estimate $\left|\varepsilon_{k}\right|<1 / 2 k$. To check (ii) note that the right-hand side of (5.4) tends to $d-1+t$, so (5.4) indeed holds for large enough $k$ for any $s \in[d-1, d-1+t)$.

5.6. Case 2: $2^{-n_{k}} \leq 2^{-q}<2^{-n_{k}+a_{k}}$. Here, the diameter of $Q$ is greater than the width of an $R_{k}^{\left(j^{\prime}\right)}$ projected onto $\ell_{k}$, but smaller than the distance of the shortest translation vector between two $R_{k}^{\left(j^{\prime}\right)}$. This is illustrated in Figure 5. 


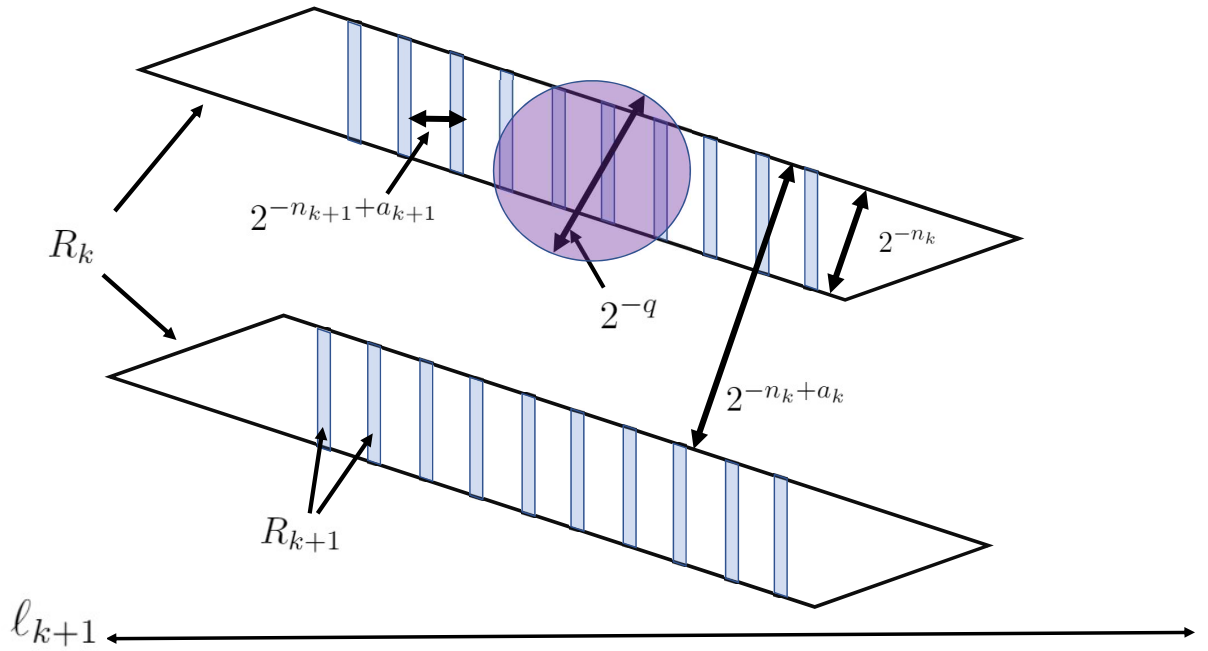

Figure 5. Positioning of $Q$ in Case 2.

Accordingly, this time we start with a similar basic estimate,

$$
\mu(Q) \leq \mu\left(R_{k+d-1}^{(i)}\right) \cdot \#\left\{R_{k+d-1}^{(i)}: R_{k+d-1}^{(i)} \cap Q \neq \emptyset\right\} .
$$

The number of $R_{k+d-1}^{(i)}$ which intersect $Q$ is bounded similarly, but this time we only take the product over the first $d-1$ terms, and we use that $Q$ can intersect at most two $R_{k}^{(i)}$.

$$
\begin{aligned}
\#\left\{R_{k+d-1}^{(i)}: R_{k+d-1}^{(i)} \cap Q \neq \emptyset\right\} & \leq 2 \prod_{j=1}^{d-1} \max _{i}\left(\#\left\{R_{k+j}^{\left(i^{\prime}\right)} \in R_{k+j-1}^{(i)}: R_{k+j}^{\left(i^{\prime}\right)} \cap Q \neq \emptyset\right\}\right) \\
& \leq 2 \prod_{j=1}^{d-1}\left[\frac{2^{-q}}{2^{-n_{k+j}+a_{k+j}}}\right] \leq 2 \prod_{j=1}^{d-1} \frac{2^{-q+1}}{2^{-n_{k+j}+a_{k+j}}} \\
& =2^{d} \prod_{j=1}^{d-1} \frac{2^{-q}}{2^{-n_{k+j}+a_{k+j}}} .
\end{aligned}
$$

Hence (5.3) is implied by the following,

$$
-m_{k+d-1}+\sum_{j=1}^{d-1}\left(n_{k+j}-a_{k+j}\right)+d \leq q(d-s-1)-2 \log _{2} q .
$$

By Lemma 4.6 iii), it suffices to show

$$
a_{k}+\left(-1+\varepsilon_{k}^{\prime}\right) n_{k} \leq q(d-s-1)-2 \log _{2} q .
$$

Notice that $d-s-1 \leq 0$, so by the hypothesis of this case $q \leq n_{k}$, it suffices to show

$$
s \leq d-\frac{a_{k}}{n_{k}}-\frac{2 \log _{2} n_{k}}{n_{k}}-\varepsilon_{k}^{\prime} .
$$

Note that this estimate is nearly identical to (5.4), hence the remainder of this argument follows mutatis mutandis. 
Acknowledgements. The authors would like to thank Richárd Balka, Kornélia Héra, András Mathé, and Pertti Mattila for their suggestions and remarks, as well as the referees for their detailed suggestions regarding the exposition. They would also like to thank the Budapest Semesters in Mathematics program for providing the framework under which this research was conducted.

\section{References}

[1] Balka, R., U. DARJi, and M. Elekes: Hausdorff and packing dimension of fibers and graphs of prevalent continuous maps. - Adv. Math. 293, 2016, 221-274.

[2] Chang, A., M. Csörnyei, K. Héra, and T. Keleti: Small unions of affine subspaces and skeletons via Baire category. - Adv. Math. 328, 2019, 801-821.

[3] Davies, R. O.: Some remarks on the Kakeya problem. - Proc. Cambridge Phil. Soc. 9, 1971, $417-421$.

[4] Eiderman, V., and M. Larsen: A "rare" plane set with Hausdorff dimension 2. arXiv:1904.09034, 2019.

[5] HÉrA, K.: Hausdorff dimension of Furstenberg-type sets associated to families of affine subspaces. - Ann. Acad. Sci. Fenn. Math. 44, 2019, 903-923.

[6] Héra, K., T. Keleti, and A. MÁthé: Hausdorff dimension of unions of affine subspaces and of Furstenberg-type sets. - J. Fractal Geom. 6, 2019, 263-284.

[7] Mattila, P.: Geometry of sets and measures in Euclidean spaces. - Cambridge Univ. Press, 1995.

[8] OBerlin, D.: Exceptional sets of projections, unions of $k$-planes and associated transforms. Israel J. Math. 202:1, 2014, 331-342.

[9] Weierstrass, K.: Über continuirliche Functionen eines reellen Arguments, die für keinen Werth des letzeren einen bestimmten Differential quotienten besitzen. - Mathematische Werke II, 71-74. Königl. Akad. Wiss., 1872.

Received 10 July $2019 \bullet$ Revised received 1 October $2020 \bullet$ Accepted 13 October 2020 